\title{
Catalogue of ISO LWS observations of asteroids ${ }^{\star}$
}

\author{
F. Hormuth ${ }^{1,2}$ and T. G. Müller ${ }^{3}$ \\ 1 Centro Astronómico Hispano Alemán, C/ Jesús Durbán Remón 2-2º 04004 Almería, Spain \\ 2 Max-Planck-Institut für Astronomie (MPIA), Königstuhl 17, 69117 Heidelberg, Germany \\ e-mail: hormuth@mpia.de \\ 3 Max-Planck-Institut für extraterrestrische Physik (MPE), Giessenbachstrasse, 85748 Garching, Germany \\ e-mail: tmueller@mpe.mpg.de
}

Received 11 October 2006 / Accepted 28 January 2009

\begin{abstract}
Context. The long wavelength spectrometer (LWS) onboard the infrared space observatory (ISO) observed the four large main-belt asteroids (1) Ceres, (2) Pallas, (4) Vesta, and (10) Hygiea multiple times. The photometric and spectroscopic data cover the wavelength range between 43 and $197 \mu \mathrm{m}$, and are a unique dataset for future investigations and detailed characterisations of these bodies. Aims. The standard ISO archive products, produced through the last post-mission LWS pipeline, were still affected by instrument artefacts. Our goal was to provide the best possible data products to exploit the full scientific potential of these observations. Methods. For all asteroid observations we analysed in detail the dark current, the calibration reference flashes, the space environment effects (glitches), memory effects, tracking influences, and various other sources of uncertainty. We performed a refined reduction of all measurements, corrected for the various effects, and re-calibrated the data. We outline the data reduction process and give an overview of the available data and the quality of the observations. We apply a thermophysical model to the flux measurements to derive far-IR based diameter and albedo values of the asteroids. The measured thermal rotational lightcurve of (4) Vesta is compared to model predictions.

Results. The catalogue of LWS (long wavelength spectrometer) observations of asteroids contains 57 manually reduced datasets, including seven non-standard observations, which as such did not have final pipeline products available before. In total, the archive now contains 11 spectral scans and 46 fixed grating measurements with a simultaneous observation at 10 key wavelengths distributed over the full LWS range. The new data products are now accessible via the ISO data archive as highly processed data products (HPDP).

Conclusions. The quality of the data products was checked against state-of-the-art thermophysical model predictions and an excellent agreement was found. The absolute photometric accuracy is better than $10 \%$. The calibrated spectra will serve as a source for future mineralogical studies of dwarf planets and dwarf planet candidates.
\end{abstract}

Key words. minor planets, asteroids - radiation mechanisms: thermal - infrared: solar system

\section{Introduction}

Between 1995 and 1998 the infrared space observatory (ISO, Kessler et al. 1996) observed more than 40 asteroids in great detail, including some complete spectra from 2 to $200 \mu \mathrm{m}$ and large samples of photometric measurements (Müller 2003; Dotto et al. 2002). The main purpose of the approximately $100 \mathrm{~h}$ of asteroid observing time was the identification of surface minerals, composition, connection to meteorites and comets, surface alteration processes, and the interpretation of taxonomic classes through the identification of mid-infrared features of well-known minerals and meteorites. Müller et al. (2005) summarised the main ISO results from the various solar system programmes and from all four ISO instruments.

The ISO Long Wavelength Spectrometer (LWS; Clegg et al. 1996) performed photometric and spectroscopic measurements in the wavelength range 43 to $197 \mu \mathrm{m}$ and opened, together with ISOPHOT, a new window for far-infrared astronomy. The details on the instrument and the data processing can be found in the ISO Handbook, Volume III (Gry et al. 2003). In total, the ISO

* Based on observations with ISO, an ESA project with instruments funded by ESA Member States (especially the PI countries: France, Germany, the Netherlands and the UK) and with the participation of ISAS and NASA.
Data Archive $\left(\mathrm{IDA}^{1}\right.$ ) contains 51 successful LWS observations of four different asteroids covering almost $18 \mathrm{~h}$ of satellite time. However, the scientific return of these programmes so far has been low. Most of the scientific questions could not be answered, mainly due to the instrumental artefacts which remained in the archived data products.

The LWS data set of asteroid observations covers a wavelength range which is not accessible from the ground. In the near future the Herschel mission will be capable of obtaining asteroid spectra between 57 and $210 \mu$ m with PACS (Photodetector Array Camera \& Spectrometer) and between 200 and $670 \mu \mathrm{m}$ with SPIRE (Spectral and Photometric Imaging Receiver), but asteroids are currently not part of the accepted key programmes. Although the ISO target list is very limited, it includes objects of high scientific interest: (1) Ceres and (4) Vesta are the targets of the Dawn ${ }^{2}$ spacecraft mission, (1) Ceres is considered as a dwarf-planet, and (2) Pallas, (4) Vesta, and (10) Hygiea are dwarf-planet candidates ${ }^{3}$. In general, it is believed that these

\footnotetext{
${ }^{1}$ http://www.iso.esac.esa.int/ida/index.html

2 http://dawn.jpl.nasa.gov/

3 Dissertatio Cvm Nvncio Sidereo III, Nr. 3, August 16, 2006, http://astro.cas.cz/nuncius/
} 
largest bodies in the main-belt are protoplanets that have remained intact since their formation.

The LWS observations comprise spectral energy distributions over the full wavelength range between 43 and $197 \mu \mathrm{m}$ with a spectral resolving power of $\sim 200$ and can be utilised to search for spectral signatures related to the asteroids' mineralogic composition. An overview of the spectroscopic features from theory and lab measurements in the infrared wavelength regime up to $25 \mu \mathrm{m}$ can be found in e.g. Salisbury (1993) and in the context of ISO in Dotto et al. (2002). Far infrared laboratory spectra of various crystalline and amorphous minerals are digitally available e.g. in the Heidelberg-Jena-St. Petersburg-Database of Optical Constants (HJPDOC) ${ }^{4}$ (Henning et al. 1999). Although these measurements are in principle sufficient to allow the analysis of the data presented in this paper, their application to solid surfaces with non-uniform grain sizes and subsurface radiation is not straightforward, rendering a mineralogic interpretation difficult.

The LWS observations also can give valuable input to models describing the thermal emission of minor bodies in our own or other solar systems, and are key ingredients to establish asteroids as calibration objects in the far infrared (e.g. Müller \& Lagerros 1998, 2002).

An effort was made to produce a homogeneously reduced set of these observations, eventually leading to a catalogue which is available through the IDA. We describe the contents of the catalogue (Sect. 2), outline the data reduction process, and give an overview of the instrumental artefacts (Sect. 3) encountered during catalogue compilation. Section 4 gives a brief overview of the catalogue structure. In Sect. 5 we discuss the quality of the final products, present diameter and albedo values derived from thermal IR data in the range 43-197 $\mu \mathrm{m}$ for the observed asteroids, and analyse the thermal lightcurve of (4) Vesta.

\section{Observations}

\subsection{Overview}

Most of the LWS asteroid observations were carried out in fixed grating mode, using the astronomical observing template (AOT) L02. This produced ten photometric measurements - one in each detector - at $46.2 \mu \mathrm{m}, 56.2 \mu \mathrm{m}, 66.1 \mu \mathrm{m}, 75.7 \mu \mathrm{m}, 84.8 \mu \mathrm{m}$, $102.4 \mu \mathrm{m}, 141.8 \mu \mathrm{m}, 160.6 \mu \mathrm{m}$, and $178.0 \mu \mathrm{m}$ (corresponding to detectors SW1 to SW5 and LW1 to LW5).

Only four full grating scans using the template L01 were performed, covering the whole spectral range from 43 to $197 \mu \mathrm{m}$ with typical SNRs of 20-30 for (10) Hygiea and 150-200 for (1) Ceres. The spectrum is composed of ten sub-spectra with the sub-spectra being generated by the grating scanning over the ten LWS detectors simultaneously. The signal-to-noise ratio was built up by taking more than one scan.

Additionally there exist 25 observations carried out in the non-standard engineering mode L99, most of them similar to L01 grating scans $(S N R \approx 150$ for (1) Ceres and $\approx 100$ for (4) Vesta). For the catalogue, only L99 on-source grating scans were considered, and no background measurements are included. The non-standard observations are characterised by experimental settings of the detector parameters (bias voltages and heater currents), an unusually high spectral sampling (13 samples per resolution element instead of four, as was the standard later), and longer integration times per grating position.

From the available L99 observations we selected seven datasets for inclusion in this catalogue. The spectral analysis

${ }^{4}$ http://www.mpia-hd.mpg.de/HJPDOC/ of this data still remains difficult, since the relative spectral response function (RSRF) of the detectors used in the calibration process is based on observations performed in the standard L01 mode. The remaining L99 data sets did not include sufficient calibration data for reliable dark current determination.

All standard observations are listed in Table 1, while the non-standard observations are summarised in Table 2. Contamination by $158 \mu \mathrm{m} \mathrm{CII} \mathrm{background} \mathrm{emission} \mathrm{is} \mathrm{indicated}$ where applicable.

A comprehensive overview of the instrument design, observing modes, and detector properties can be found in the LWS handbook (Gry et al. 2003).

\subsection{Observation elements}

Each observation consists not only of flux measurements of the target itself, but also includes calibration data, typically split in two blocks at the beginning and end. By placing the FabryPerót spectrometer into the beam and intentionally misaligning the etalons, light from the source did not reach the detectors, eventually resulting in a dark current measurement. Long observations, and especially the engineering mode L99 data, contain intermittent additional dark current measurements.

Since the responsivity of the detectors is not constant, but usually drifting upwards over time due to impacts of charged particles, the actual responsivity of each detector had to be determined for each observation separately. This was done by flashing the detectors with internal illuminators while light from the source was again blocked with the Fabry-Perót. This allowed to quantify responsivity changes during the course of a single observation, and to apply the so-called "responsivity drift correction".

Absolute responsivity calibration was performed by comparing the responsivities measured at the time of the observation to the ones measured during observations of Uranus, the primary LWS calibrator.

For the target source flux measurements, the Fabry-Perót was removed from the beam. Depending on the observation template and requested wavelength coverage, the grating remained either at a fixed angle or was moved to scan the desired spectral range. This could be done in unidirectional mode, where the grating angle constantly in- or decreased and was reset to its start position before each scan repetition. Alternatively, the observation could be performed in bidirectional mode, with the grating angle increasing and then decreasing with the same rate during the measurement. This is referred to as the up- and down-scan, where "up" and "down" does not correspond to an increase or decrease of the observed wavelength, but of the grating position encoder value. In fact, during an upscan the observing wavelength decreased, and increased during downscans.

\section{Data reduction}

\subsection{Instrumental artefacts}

The most prominent artefacts, common to all ISO instruments, are "glitches", sudden increases of the measured flux due to charged particles hitting a detector or parts of the readout electronics. The initial sudden signal increase is sometimes followed by a "glitch tail", a long-lived exponential decay. Though glitches can be recognised and filtered out automatically to some extent, glitch remnants are present in all observations throughout the archive, regardless of instrument or observing mode. Glitches do not only occur during object flux measurements, 
Table 1. Overview of standard LWS asteroid observations in our catalogue. For the on-source pointings the distance to the asteroid $\Delta$, the Sunasteroid distance $r$, and the phase angle $\alpha$ are given, based on MPC (minor planet center) ephemerides. The TDT number is a unique identifer, allowing one to find the observation in the archive.

\begin{tabular}{|c|c|c|c|c|c|c|c|c|c|}
\hline \multicolumn{2}{|c|}{ Object } & \multirow{3}{*}{$\begin{array}{l}\text { TDT } \\
09300401\end{array}$} & \multicolumn{2}{|c|}{ Mid-observation UTC } & \multirow{2}{*}{$\begin{array}{l}\text { AOT } \\
\text { L02 }\end{array}$} & \multirow{2}{*}{$\begin{array}{l}\Delta[\mathrm{AU}] \\
2.673\end{array}$} & \multirow{2}{*}{$\begin{array}{l}r \text { [AU] } \\
2.695\end{array}$} & \multicolumn{2}{|c|}{$\begin{array}{cc}\alpha\left[^{\circ}\right] & \text { Remarks } \\
\end{array}$} \\
\hline (1) & Ceres & & 1996-02-18 & $04: 31: 18$ & & & & 21.2 & \\
\hline & & & 1996-02-18 & $15: 15: 23$ & L02 & \multicolumn{4}{|c|}{ (off-source reference measurement for TDT 09300401) } \\
\hline & & 10500402 & 1996-03-01 & $05: 22: 22$ & L02 & 2.520 & 2.705 & 21.5 & \\
\hline & & 11900214 & 1996-03-15 & $02: 42: 57$ & L02 & 2.344 & 2.715 & 21.1 & \\
\hline & & 11905611 & $1996-03-15$ & $16: 54: 46$ & L02 & \multicolumn{4}{|c|}{ (off-source reference measurement for TDT 11900214) } \\
\hline & & 12600114 & 1996-03-22 & 02:03:54 & L02 & 2.258 & 2.721 & 20.5 & \\
\hline & & 25800302 & 1996-07-31 & $19: 30: 27$ & L02 & 2.258 & 2.823 & 19.2 & \\
\hline & & 25805903 & 1996-08-01 & 08:55:54 & L02 & \multicolumn{4}{|c|}{ (off-source reference measurement for TDT 25800302) } \\
\hline & & 26500301 & 1996-08-07 & $19: 10: 27$ & L02 & 2.351 & 2.828 & 20.0 & \\
\hline & & 26505602 & 1996-08-08 & $09: 32: 38$ & L02 & \multicolumn{4}{|c|}{ (off-source reference measurement for TDT 26500301) } \\
\hline & & 32100204 & 1996-10-02 & $15: 31: 35$ & L02 & 3.126 & 2.868 & 18.6 & \\
\hline & & 32103506 & 1996-10-03 & 03:52:44 & L02 & \multicolumn{4}{|c|}{ (off-source reference measurement for TDT 32100204) } \\
\hline & & 53802209 & 1997-05-07 & 12:16:09 & L02 & 3.112 & 2.971 & 18.9 & \\
\hline & & 57902409 & 1997-06-17 & 09:56:26 & L02 & 2.575 & 2.978 & 19.4 & \\
\hline & & 59401908 & 1997-07-02 & 06:42:05 & L02 & 2.391 & 2.980 & 17.9 & \\
\hline & & 72001901 & 1997-11-05 & 01:10:04 & L02 & 2.522 & 2.973 & 18.6 & \\
\hline & & 74803304 & 1997-12-03 & 03:04:58 & L02 & 2.901 & 2.966 & 19.3 & \\
\hline & & 74803403 & 1997-12-03 & 08:11:59 & L01 & 2.904 & 2.966 & 19.3 & contains warmup artefacts \\
\hline & & 75502902 & $1997-12-10$ & 00:30:08 & L01 & \multicolumn{4}{|c|}{ (off-source reference measurement for TDTs $74803 \ldots$... } \\
\hline & & 75503003 & $1997-12-10$ & 01:38:00 & L02 & 2.994 & 2.964 & 19.0 & \\
\hline & & 76200502 & $1997-12-16$ & $13: 29: 15$ & L02 & 3.081 & 2.962 & 18.6 & \\
\hline & & 76903102 & $1997-12-24$ & 01:08:50 & L02 & 3.177 & 2.960 & 18.0 & \\
\hline & & 76903203 & $1997-12-24$ & 02:17:12 & L01 & 3.178 & 2.960 & 18.0 & \\
\hline \multirow[t]{8}{*}{ (2) } & Pallas & 23000306 & 1996-07-03 & 21:24:01 & L02 & 2.497 & 2.823 & 20.9 & \\
\hline & & 23002907 & 1996-07-04 & $12: 01: 22$ & L02 & \multicolumn{4}{|c|}{ (off-source reference measurement for TDT 23000306) } \\
\hline & & 25100202 & $1996-07-24$ & $19: 49: 12$ & L02 & 2.780 & 2.871 & 20.6 & \\
\hline & & 25103603 & $1996-07-25$ & 09:58:26 & L02 & \multicolumn{4}{|c|}{ (off-source reference measurement for TDT 25100202) } \\
\hline & & 26500503 & 1996-08-07 & 19:43:17 & L02 & 2.967 & 2.903 & 19.9 & \\
\hline & & 26505204 & 1996-08-08 & 07:27:57 & L02 & \multicolumn{4}{|c|}{ (off-source reference measurement for TDT 26500503) } \\
\hline & & 27200203 & 1996-08-14 & 18:19:41 & L02 & 3.057 & 2.919 & 19.3 & \\
\hline & & 27202004 & $1996-08-15$ & 03:27:01 & L02 & \multicolumn{4}{|c|}{ (off-source reference measurement for TDT 27200203) } \\
\hline \multirow[t]{15}{*}{ (4) } & Vesta & 24402202 & $1996-07-17$ & $23: 20: 20$ & L02 & 1.593 & 2.149 & 26.6 & \\
\hline & & 24404603 & 1996-07-18 & $06: 29: 49$ & L02 & (off-sou & e referen & e meas & rement for TDT 24 \\
\hline & & 80500101 & 1998-01-28 & $10: 36: 25$ & L02 & 2.566 & 2.549 & 22.2 & \\
\hline & & 80500104 & $1998-01-28$ & 11:02:50 & L02 & 2.566 & 2.549 & 22.2 & \\
\hline & & 80500107 & $1998-01-28$ & $11: 29: 15$ & L02 & 2.567 & 2.549 & 22.2 & \\
\hline & & 80500110 & $1998-01-28$ & $11: 55: 40$ & L02 & 2.567 & 2.549 & 22.2 & \\
\hline & & 80500113 & 1998-01-28 & $12: 22: 05$ & L02 & 2.567 & 2.549 & 22.2 & \\
\hline & & 80500116 & $1998-01-28$ & $12: 48: 30$ & L02 & 2.567 & 2.549 & 22.2 & \\
\hline & & 80500119 & $1998-01-28$ & $13: 14: 55$ & L02 & 2.568 & 2.549 & 22.2 & \\
\hline & & 80500122 & 1998-01-28 & $13: 41: 20$ & L02 & 2.568 & 2.549 & 22.2 & \\
\hline & & 80500125 & 1998-01-28 & 14:07:45 & L02 & 2.568 & 2.549 & 22.2 & \\
\hline & & 80500128 & $1998-01-28$ & 14:34:10 & L02 & 2.568 & 2.549 & 22.2 & \\
\hline & & 80500131 & $1998-01-28$ & $15: 00: 35$ & L02 & 2.569 & 2.549 & 22.2 & \\
\hline & & 80500134 & 1998-01-28 & $15: 27: 00$ & L02 & 2.569 & 2.549 & 22.2 & \\
\hline & & 80500137 & $1998-01-28$ & $15: 53: 25$ & L02 & 2.569 & 2.549 & 22.2 & \\
\hline (10) & Hygiea & 83201702 & $1998-02-24$ & 22:08:03 & L01 & 3.179 & 3.444 & 16.6 & CII background emission \\
\hline & & 83201803 & 1998-02-24 & $22: 44: 05$ & L02 & 3.179 & 3.444 & 16.6 & \\
\hline & & 84801302 & 1998-03-12 & 16:13:06 & L02 & 3.402 & 3.434 & 16.7 & \\
\hline & & 85303402 & 1998-03-17 & 16:09:46 & L02 & 3.472 & 3.430 & 16.6 & \\
\hline
\end{tabular}

but also during the calibration, i.e. dark current and responsivity measurements, and adversely affect the accuracy and reliability of the actual observation. Special attention was therefore paid to the manual removal of glitch remnants in each observation contained in the presented catalogue.

Other frequently observed instrumental artefacts are fringes in the spectra in the case of extended sources or off-axis observations, spurious spectral features due to uncertainties in the spectral response function used in the calibration process, and memory effects, i.e. changes of detector response depending on the illumination history.
Observations towards the end of the satellite mission were affected by so-called "warm-up effects", visible as broad spectral features in some detectors. This was presumably caused by periodic breaks of the liquid helium film in the vicinity of the LWS strap location as the satellite's helium tank came close to exhaustion. From the observations contained in this catalogue, this affects one L01 type observation of (1) Ceres taken in December 1997, several months before ISO's liquid helium depletion in April 1998.

A thorough description of caveats and unexpected effects concerning LWS data can be found in Chapter 6 of the LWS 
Table 2. Overview of L99 asteroid observations in our catalogue. All observations are L01-like scans covering the full wavelength range.

\begin{tabular}{|c|c|c|c|c|c|c|c|}
\hline \multicolumn{2}{|c|}{ Object } & TDT & Mid-observation UTC & $\Delta[\mathrm{AU}]$ & $r[\mathrm{AU}]$ & $\alpha\left[^{\circ}\right]$ & Remarks \\
\hline \multirow[t]{4}{*}{ (1) } & Ceres & 07500601 & 1996-01-31 07:52:11 & 2.891 & 2.682 & 19.9 & CII background emission \\
\hline & & 07500701 & 1996-01-31 10:10:56 & 2.890 & 2.682 & 19.9 & CII background emission \\
\hline & & 07501301 & 1996-01-31 16:08:13 & 2.887 & 2.682 & 19.9 & $\begin{array}{l}\text { CII background emission, } \\
\text { non-standard bias voltages }\end{array}$ \\
\hline & & 07501401 & 1996-01-31 18:32:03 & 2.886 & 2.682 & 20.0 & $\begin{array}{l}\text { CII background emission, } \\
\text { non-standard bias voltages }\end{array}$ \\
\hline \multirow[t]{3}{*}{ (4) } & Vesta & 05500201 & 1996-01-11 07:10:35 & 2.313 & 2.250 & 24.8 & non-standard bias voltages \\
\hline & & 05500301 & 1996-01-11 08:54:29 & 2.312 & 2.250 & 24.9 & non-standard bias voltages \\
\hline & & 05500401 & 1996-01-11 10:39:34 & 2.311 & 2.250 & 24.9 & non-standard bias voltages \\
\hline
\end{tabular}

handbook (Gry et al. 2003). All datasets included in this catalogue were checked for the presence of the artefacts described above and data quality was quantified via a set of well-defined flags. The criteria for setting these flags and the results of this quality check can be found in the technical documentation accompanying the catalogue (Hormuth \& Müller 2006).

\subsection{Data reduction steps}

Data reduction was performed with the available standard reduction packages LIA (LWS Interactive Analysis) Version 10.2 and ISAP (ISO Spectral Analysis Package) Version 2.2. Additional custom IDL-procedures were used for manual editing of the data at the SPD (Standard Processed Data) level, and the generation of the final FITS and ASCII files. The SPD data is preprocessed in the sense that raw detector readouts have been converted into photocurrents, saturated readouts flagged, and strong glitches automatically identified and removed. Housekeeping data not necessary for further data reduction has been removed, but dark current and responsivity measurements have been preserved. In the following we briefly outline the order of reduction steps and give the names of the corresponding LIA procedures where applicable.

In a first step, the pointing coordinates of each observation were checked against the ephemeris of the observed asteroid to detect pointing problems. The maximum difference between asteroid position and telescope pointing was found to be 2 '. 5 , much smaller than the LWS beam profile (see Lloyd 2003). The reduction process started at SPD level with a visual inspection of the data in the time domain. Photometric L02 observations were manually de-glitched at this stage, capturing minor glitches not automatically flagged during generation of the SPD data. The available pipeline data of grating scans was additionally inspected with ISAP to get a quick overview of the available scans and scan directions.

Next, dark current determination and subtraction were performed using the LIA routine IA_DARK, followed by the absolute responsivity correction with IA_ABSCORR. These interactive routines allow visual inspection and manual editing of the dark current measurements and the internal calibration source observations. Deglitching of this data and the removal of measurements obviously affected by memory effects is critical, since any artefacts in the calibration data will affect the accuracy and reliability of the whole observation. The LIA routine SHORT_AAL was used to create ISAP-compatible output spectra in the LWS AutoAnalysis format, and to convert to $\mathrm{W} / \mathrm{cm}^{2} / \mu \mathrm{m}$, the flux unit used for all LWS observations. This routine automatically strips all calibration data and leaves only the basic information needed for further scientific analysis, e.g. wavelength, flux, detector number, and scan direction.
In the case of photometric L02 observations, the data was now averaged per detector to create the final FITS product.

In the case of grating scans a first round of manual deglitching was performed, probably the most time consuming step in the reduction process. Relative responsivity correction was achieved by comparing the mean flux in each detector and scan to the mean flux level of all scans together. The derived gains were then used to bring all scans to the same flux level - separately for each detector and scan direction.

After manual removal of remaining glitches, the data was averaged per detector with a bin width of typically $0.06 \mu \mathrm{m}$, corresponding to an oversampling of four with respect to the spectral resolution. In the case of bidirectional scans the data was now averaged over the scan directions. Especially at longer wavelengths some spectra clearly showed fringing, probably caused by background emission. The affected detectors were defringed within ISAP, using only the averaged spectrum for fringe detection and defringing to get the best possible signal-to-noise ratio for the fringe fitting (in the case of bidirectional data).

\section{Data products and access to the catalogue}

The ISO Data Archive (IDA) $)^{5}$ contains spectroscopic, imaging, photometric, and polarimetric measurements of more than 40 different asteroids at wavelengths between 2 and $240 \mu \mathrm{m}$. A versatile Java based web-interface allows to extract observational data in various formats, ranging from unprocessed raw data and standard pipeline processed measurements up to manually reprocessed high quality data products.

All data files associated with this catalogue can be retrieved by searching for the specific TDT $^{6}$ number of a measurement as given in Tables 1 and 2, by searching for all LWS observations of a given asteroid, or by searching for LWS asteroid observations in general. The highly processed data products, including overview plots, quality flags, and catalogue documentation can be obtained via the HPDP button to the left of each entry in the search result window of the archive interface.

The catalogue documentation (Hormuth \& Müller 2006), giving a thorough description of the data reduction steps, is also separately available from the list of all HPDP data sets ${ }^{7}$.

In Figs. 1 and 2 we show example logarithmic plots for all observing modes. Different colours have been chosen to allow one to distinguish overlapping measurements by different detectors. The error bars in Fig. 1 include measurement and calibration errors, incuding a 5\% uncertainty attributed to the Uranus models used for absolute flux calibration. The slight mismatches between measurements from different detectors at the

\footnotetext{
5 Accessible via http://iso.esac.esa.int

6 "Target Dedicated Time", a unique identifier for ISO observations.

7 http://iso.esac.esa.int/ida/hpdp.php
} 



Fig. 1. Examples for L02 observations. Left: fixed grating observation of (2) Pallas. Right: short grating scan observation of (10) Hygiea.

same wavelengths in Fig. 2 are caused by memory effects of individual detectors.

The data sets are available both as FITS tables, compatible with the ISAP software package, and as plain ASCII tables. Please refer to the catalogue documentation for information about individual FITS header keywords and table columns.

\section{Discussion}

\subsection{Quality of the catalogue products}

In order to study the reliability of the photometric L02 data in the catalogue, we compared the measured flux with predictions calculated by means of the thermophysical model (TPM; e.g. Lagerros 1998, and references therein). For Table 3 we calculated model fluxes for the specified targets and epochs and analysed the observation-to-model ratios. Measurements affected by detector warm-up artefacts or abnormally high dark current were not included in this quality check.

The general agreement between the photometric observations (L02) and models (Table 3) is better than 5\% for Ceres, Pallas, and Vesta and better than $30 \%$ for Hygiea. The higher uncertainties are due to dark current effects at the low flux levels. The discrepancy for Hygiea is not surprising. Due to the lack of suitable good quality thermal data the thermophysical model parameters are also very poor, and Hygiea is considered as a low
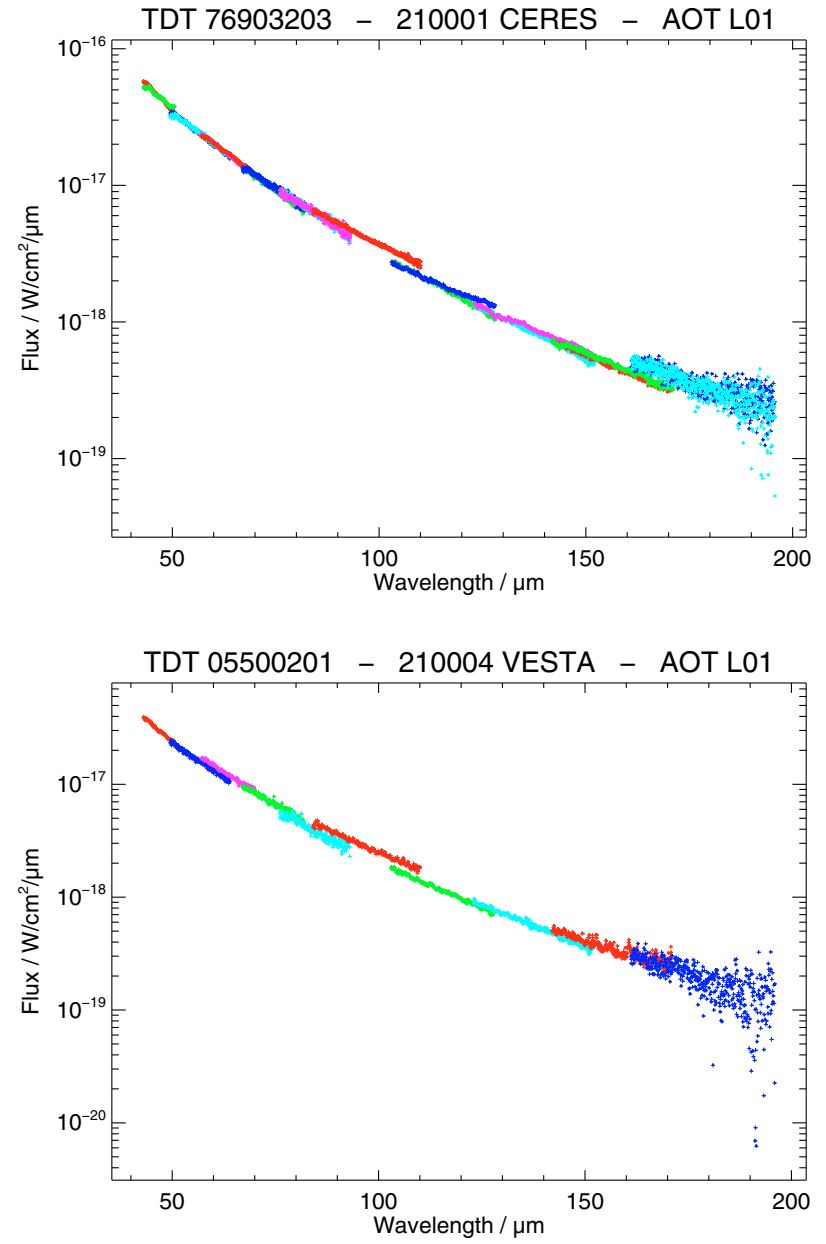

Fig. 2. Examples of full wavelength range scans. Left: standard L01 observation of (1) Ceres. Right: engineering mode L99 observation of (4) Vesta.

Table 3. Ratios between LWS 02 observations and TPM predictions, weighted mean values and standard deviations per detector (SW1, 2, 3, 4,5 and LW1, 2, 3) and per asteroid. The photometry of the LW4 and LW5 detectors is less reliable due to dark current problems.

\begin{tabular}{rcccc}
\hline \hline$\lambda$ & $\begin{array}{c}\text { (1) Ceres } \\
\text { 11 Obs. }\end{array}$ & $\begin{array}{c}\text { (2) Pallas } \\
\text { 4 Obs. } \\
\text { Obs/Mod }\end{array}$ & $\begin{array}{c}\text { (4) Vesta } \\
\text { 14 Obs. } \\
\text { Obs/Mod }\end{array}$ & $\begin{array}{c}\text { (10) Hygiea } \\
\text { 2 Obs. } \\
\text { Obs/Mod }\end{array}$ \\
\hline 46.4 & $1.04 \pm 0.05$ & $1.00 \pm 0.02$ & $1.02 \pm 0.04$ & $1.21 \pm 0.05$ \\
56.4 & $1.01 \pm 0.03$ & $1.06 \pm 0.02$ & $1.01 \pm 0.04$ & $1.25 \pm 0.10$ \\
66.3 & $1.04 \pm 0.02$ & $1.10 \pm 0.04$ & $1.02 \pm 0.04$ & $1.23 \pm 0.15$ \\
75.9 & $1.02 \pm 0.03$ & $1.02 \pm 0.03$ & $1.04 \pm 0.04$ & $1.24 \pm 0.12$ \\
85.0 & $1.01 \pm 0.04$ & $1.02 \pm 0.11$ & $1.00 \pm 0.07$ & $1.39 \pm 0.10$ \\
102.9 & $1.09 \pm 0.04$ & $1.13 \pm 0.04$ & $1.24 \pm 0.06$ & $1.40 \pm 0.26$ \\
122.7 & $0.96 \pm 0.05$ & $0.95 \pm 0.03$ & $1.07 \pm 0.05$ & $1.30 \pm 0.07$ \\
142.2 & $0.89 \pm 0.04$ & $0.84 \pm 0.07$ & $1.10 \pm 0.11$ & $1.33 \pm 0.04$ \\
161.0 & $0.99 \pm 0.05$ & $0.99 \pm 0.04$ & - & - \\
178.4 & $1.01 \pm 0.10$ & - & - & - \\
\hline Mean & $1.01 \pm 0.05$ & $1.03 \pm 0.08$ & $1.05 \pm 0.08$ & $1.27 \pm 0.14$ \\
\hline
\end{tabular}

quality far-IR calibrator (Müller \& Lagerros 1998). As a rule of thumb, the absolute flux error is better than $10 \%$ for targets brighter than about $10 \mathrm{Jy}$.

The high quality of the spectral scans can be seen in the smooth and well-connected curves of the archive browse images (see Fig. 2). For mineralogic studies it might still be useful 
Table 4. Object specific TPM input parameters. The $\lambda_{\text {ecl }}$ and $\beta_{\text {ecl }}$ values are the ecliptic coordinates of the spin vector direction, with $\beta_{\text {ecl }}$ counted from the equator.

\begin{tabular}{ccrcl}
\hline \hline Object & {$[H, G]$} & $P_{\text {sid }}[\mathrm{h}]$ & \multicolumn{1}{c}{$\lambda_{\text {ecl }}, \beta_{\text {ecl }}$} & Source of shape \& spin vector \\
\hline (1) Ceres & {$[3.28,0.05]^{1}$} & 9.074184 & $331.51^{\circ},+77.88^{\circ}$ & HST observations ${ }^{4}$ \\
(2) Pallas & {$[4.13,0.16]^{2}$} & 7.813225 & $34.80^{\circ},-11.75^{\circ}$ & Lightcurve inversion techniques ${ }^{5,6}$ \\
(4) Vesta & {$[3.20,0.34]^{3}$} & 5.342129 & $319.45^{\circ},+59.23^{\circ}$ & HST observations \\
(10) Hygiea & {$[5.43,0.07]^{2}$} & 27.623265 & $117.01^{\circ},-28.53^{\circ}$ & Lightcurve inversion techniques $^{8}$ \\
\hline
\end{tabular}

${ }^{1}$ Lagerkvist et al. (1992); ${ }^{2}$ Lagerkvist et al. (2001); ${ }^{3}$ Müller \& Lagerros (1998); ${ }^{4}$ Thomas et al. (2005); ${ }^{5}$ M. Kaasalainen, priv. comm. 22/Sep./2005; ${ }^{6}$ Torppa et al. (2003); ${ }^{7}$ Thomas et al. (1997); ${ }^{8}$ M. Kaasalainen, priv. comm. 31/Jan./2006;

to investigate the up- and down-scans separately to confirm whether certain low-level, broad-band structures in the scans are reliable or not. Low level features extending over two or more detectors as well as structures close to pronounced RSRF changes have to be analysed carefully: the detectors do not always behave in exactly the same way and LWS should be considered as 10 individual spectrometers, one per detector.

\subsection{Diameter and albedo calculations}

Direct size measurements, even for the large main-belt asteroids, are still difficult. Up to now, only a small sample has been resolved via imaging techniques from space (HST, see Dotto et al. 2002, and references therein) and adaptive optics techniques from large ground-based telescopes (e.g. Marchis et al. 2006). Occultation observations performed by well-organised groups of amateur astronomers also reveal direct sizes, expressed in chord maps which represent the cross section of the asteroid at the time of the occultation (e.g. Millis \& Dunham 1989) or http : //www. psi.edu/pds/resource/occ.html for more recent results). But the largest number of asteroid sizes originate from thermal infrared observations (e.g. Tedesco et al. 2002a,b) via radiometric methods i.e., an indirect method which relies on model assumptions.

We used a well-established thermophysical model (TPM, Lagerros 1996, 1997, 1998) to derive absolute effective sizes and albedos for our 4 asteroids from the new L02 data sets.

This model allows us to use state-of-the-art shape models together with corresponding spin axis solutions derived from lightcurve inversion techniques (e.g. Kaasalainen et al. 2002). As part of the energy equation for the asteroids the amount of reflected sunlight is described via the $H-G$ magnitude system (Bowell et al. 1989). The rest of the solar energy is absorbed and re-emitted as thermal emission. Knowing the solar insolation together with the disk-integrated thermal emission allows us then to solve the energy equation for the asteroid's effective size and albedo (e.g. Harris \& Lagerros 2002). The true illumination and observing geometries are taken into account in the TPM calculations. The surface roughness is described by the rms values of the surface slopes $(\rho)$ and the fraction of the surface covered by craters $(f)$. For the surface roughness, as well as for the thermal properties used to calculate the heat conduction into lower layers of the surface, we used "default properties" ( $\rho=0.7, f=0.6$, $\Gamma=15 \mathrm{~J} \mathrm{~m}^{-2} \mathrm{~s}^{-0.5} \mathrm{~K}^{-1}$ ) as described by Müller et al. (1999). The emissivity models were taken from Müller \& Lagerros (1998): a standard wavelength-dependent emissivity model for Ceres, Pallas, ad Hygiea and a special one for Vesta where lower emissivities in the submm/mm range were found.

The TPM with the "default thermal properties" has been tested and validated extensively in the context of ISO for large, regolith-covered main-belt-asteroids (Müller \& Lagerros 2002).
Table 5. Radiometric diameter and albedo values, derived from the LWS L02 measurements.

\begin{tabular}{lccc}
\hline \hline Object & $D_{\text {eff }}[\mathrm{km}]$ & $p_{V}$ & $N$ \\
\hline (1) Ceres & $959.6 \pm 16.9$ & $0.096 \pm 0.003$ & 55 \\
(2) Pallas & $534.4 \pm 14.7$ & $0.142 \pm 0.009$ & 20 \\
(4) Vesta & $548.5 \pm 12.5$ & $0.317 \pm 0.015$ & 70 \\
(10) Hygiea & $469.3 \pm 26.5$ & $0.056 \pm 0.006$ & 20 \\
\hline
\end{tabular}

The surface roughness and heat conduction properties are less important for our far-IR analysis since they mainly influence the mid-IR radiation at the Wien-part of the spectral energy distribution (e.g. Müller 2002, Figs. 2 and 3).

Asteroid-specific model input parameters and the corresponding references are listed in Table 4.

Our derived diameter and albedo values are shown in Table 5. The diameters are effective diameters of a sphere of equal volume. It can be considered as a "scaling factor" for shape models which lack absolute size information. These values are the weighted mean values, based on the photometrically more reliable short wavelength detectors $(46.4$ to $85.0 \mu \mathrm{m})$. We also excluded the data which are flagged as being affected by high dark currents or high glitch rates. In the table we give the full standard deviation of all $N$ radiometric solutions to account for the fact that different observations see the asteroid under different observing and illumination geometries.

These diameter and albedo values are the first ones that are derived purely from far-IR observations. Nevertheless, the results agree very well with the published values so far.

The most accurate values for the diameter of (1) Ceres come from HST observations (Thomas et al. 2005) and adaptive optics (AO) observations using the Keck II telescope (Carry et al. 2008). The HST observations led to an oblate spheroid with axes of $a=b=487.3 \mathrm{~km}$ and $c=454.7 \mathrm{~km}$, resulting in an effective diameter of $2 \times(a \times b \times c)^{1 / 3}=952.4 \mathrm{~km}$. The AO data confirmed the oblate spheroid, but with slightly different values $(a=b=479.7 \pm 2.3 \mathrm{~km}, c=444.4 \pm 2.1 \mathrm{~km})$ and an effective diameter of $935.3 \mathrm{~km}$. Our result is derived from very limited viewing geometries, but it is in excellent agreement with the HST results. In this context it is interesting to note that the diameter derived from IRAS observations at 12, 25, 60 and $100 \mu \mathrm{m}$ was $848.40 \pm 19.7 \mathrm{~km}$ (Tedesco et al. 2002b). Also our albedo value agrees very well with the 0.0936 derived via stellar occultation techniques (Shevchenko \& Tedesco 2006).

(2) Pallas: Tedesco et al. (2002b) obtained from IRAS data values of $498.07 \pm 18.8 \mathrm{~km}$ and a geometric albedo of $0.1587 \pm$ 0.013 . A combination of speckle, occultation and lightcurve observations of (2) Pallas lead to an ellipsoidal shape with dimensions of $574 \pm 10 \times 526 \pm 3 \times 501 \pm 2 \mathrm{~km}$ and a mean diameter of $533 \pm 6 \mathrm{~km}$ (Dunham et al. 1990). A recent high angular resolution adaptive optics study by Carry et al. (2007) resulted in an 
absolute effective size of $519.5 \mathrm{~km}$ (semi-major ellipsoidal axes values of $276 \times 256 \times 248 \mathrm{~km}, \pm 10 \mathrm{~km})$. Drummond \& Christou (2008) found $494 \pm 57 \mathrm{~km}$ based on another set of AO observations. The various results show that there are still significant uncertainties in the overall shape and size values, but the derived effective sizes agree within the given errorbars with our radiometric diameter. Therefore, we believe that our albedo of 0.142 is the most reliable value so far published for Pallas.

(4) Vesta has a very complex surface with large albedo variations on the surface (Binzel et al. 1997). Our diameter value is about $4 \%$ larger than the effective diameter from HST observations (axes 289, 280, and $229 \pm 5 \mathrm{~km}$; $D_{\text {eff }}=529.2 \mathrm{~km}$; Thomas et al. (1997)) and about 7\% larger than the mean triaxial ellipsoid solution with $563 \pm 5 \times 534 \pm 5 \times 442 \pm 7 \mathrm{~km}$ $\left(2 \cdot(a \cdot b \cdot c)^{1 / 3}=510.3 \pm 5.6 \mathrm{~km}\right)$ given by Drummond $\&$ Christou (2008). This is outside the given rms-scatter from our 70 measurements, which might be an indication that the special emissivity model for Vesta (Müller \& Lagerros 1998) needs a small adjustment for this wavelength range. It looks like the emissivity drop seen by Redman et al. $(1992,1998)$ already occurs at shorter wavelengths, below $50 \mu \mathrm{m}$. Almost all LWS observations of Vesta were taken on one day (revolution 805) under very similar aspect angles. This might also influence the outcome of the TPM technique which works best when combining data from different wavelengths, phase angles, rotational phases and aspect angles (Müller \& Lagerros 2002). Shevchenko $\&$ Tedesco (2006) derived an albedo of $p_{H}=0.370$ from occultation measurements, while we obtained a radiometric value of $p_{V}=0.32 \pm 0.02$; again this discrepancy might be explained by emissivity effects or the limited aspect angle range. The albedo value in Table 5 agrees very well with previous studies by Müller \& Lagerros (1998) $\left(p_{V}=0.33\right)$ based on a set of thermal observations from mid-IR to mm-wavelengths.

Our result for (10) Hygiea deviates significantly from previous studies: Tedesco et al. (2002b) found values of $407.12 \pm$ $6.8 \mathrm{~km}$ and $p_{\mathrm{V}}=0.0717 \pm 0.002$. Müller \& Lagerros (1998) gave $429.9 \mathrm{~km}$ and 0.066 . But Hygiea's shape model is not that well defined due to a poor lightcurve coverage (in rotational phases) and some very low quality lightcurves (Kaasalainen, priv. comm.). There might also be a second pole solution at around $\lambda_{\mathrm{ecl}}=300^{\circ}$ (instead of the $117^{\circ}$ used here). Neither using the second pole solutions nor using a spherical shape model lower the predicted size values (or the standard deviations) significantly. One possibility for the discrepancies in the diameters could be emissivity issues. If Hygiea's far-IR emissivity is lower then the corresponding diameter would also be smaller (and the albedo slightly larger). But the data quality and the very limited aspect angle range (all LWS observations were taken within 20 days) are not sufficient to draw firm conclusions. Especially when looking at the best quality direct size measurement by Ragazzoni et al. (2000): they gave an effective diameter of $444 \pm 35 \mathrm{~km}$ (with an axis-ratio of $a / b=1.11$ ), based on speckle techniques. Our radiometric results are still well within these error bars.

\subsection{Far-IR thermal lightcurve of (4) Vesta in the range 45-105 $\mu \mathrm{m}$}

The visual lightcurve of (4) Vesta is dominated by the influence of the albedo variations (Degewij et al. 1979). Standard lightcurve inversion techniques failed to produce a reliable shape model (Kaasalainen, priv. comm.), but high resolution HST imaging allowed a solution for the shape and spin vector (Thomas et al. 1997). Redman et al. (1992) found that the



Fig. 3. The observed (dots with error bars) and the predicted thermal lightcurve (continuous line) of (4) Vesta at $102.3 \mu \mathrm{m}$ (detector LW1).

$1 \mathrm{~mm}$ light-curve is apparently dominated by the triaxial shape, without any significant contributions from the optical albedo spots. Müller \& Barnes (2007) showed that at even longer wavelengths (around $3.2 \mathrm{~mm}$ ) the mm-lightcurve follows the shapeintroduced variations for a large fraction of the rotational period. They also demonstrated that the rotational phases with clear deviations are connected to structures (e.g., the Olbers feature) which are visible in the HST images of (4) Vesta (Binzel et al. 1997).

Before we combined TPM lightcurve predictions with the observed fluxes, we explored the influence of TPM input parameters on the thermal lightcurve amplitude and phase for the given wavelength range and aspect angles. It turned out that neither the thermal inertia (the range between 0 and $50 \mathrm{~J} \mathrm{~m}^{-2} \mathrm{~s}^{-0.5} \mathrm{~K}^{-1}$ was considered) nor the beaming parameter, describing various roughness scenarios, influenced the predictions for our case significantly. The predicted lightcurve amplitudes varied between 5-6\% (peak-to-peak) and the lightcurve phases only by a few minutes between the low and high thermal inertia predictions. Our data sets were therefore not sufficient to confine any of these parameters.

The Vesta L02 observations from revolution 805 (28-Jan.1998) were taken over a period of about $6 \mathrm{~h}$, covering slightly more than one full rotation period of $5.34 \mathrm{~h}$. On the basis of the shape and spin-vector solution given in Table 4 and the diameter and albedo results from Table 5 we predicted the thermal lightcurves at the L02 key-wavelengths for the LWS observations (note that the reference time frame is that of the ISO satellite and not the asteroid-intrinsic one).

Figure 3 shows the observations at $102.3 \mu \mathrm{m}$ together with the model prediction on an absolute flux and time scale. Figure 4 shows the combined data below $105 \mu \mathrm{m}$, first normalised per detector over the full observing period and then averaged for a given rotational phase angle. The quality of the data from the longer wavelength channels beyond $105 \mu \mathrm{m}$ was not sufficient for this kind of analysis.

Both figures show that the observations follow the model predictions. The errorbars in Fig. 4 correspond to the error of the mean values plus a contribution from the flux uncertainty in these detectors. This figure combines in total 78 independent measurements $(6$ detectors $\times 13$ measurements at different 


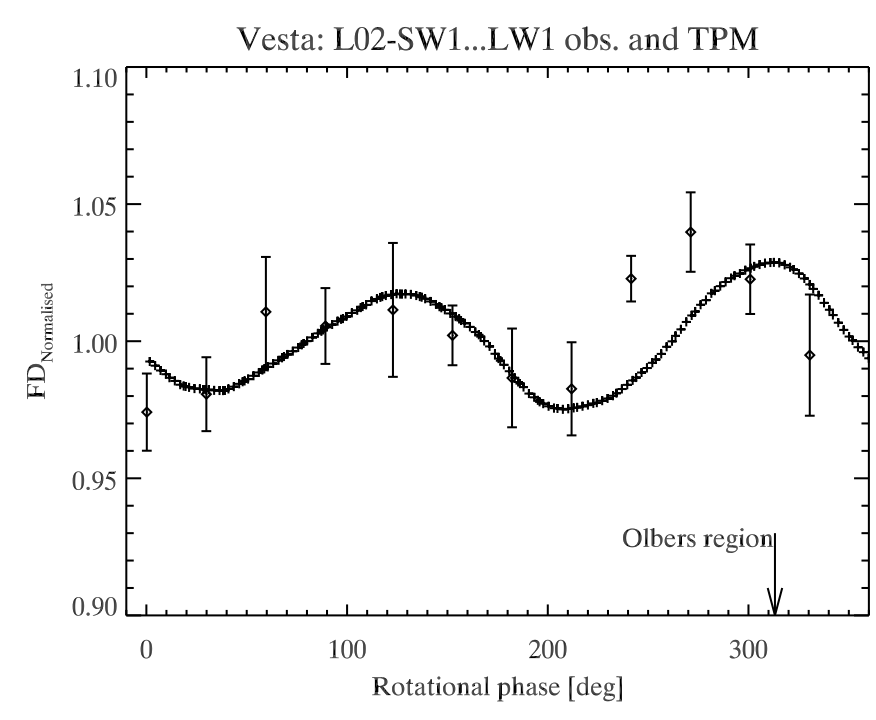

Fig. 4. The observed (dots with error bars), normalised and averaged over five detectors (SW1, SW2, SW3, SW4, SW5 and LW1) and the predicted thermal lightcurve (continuous line) of (4) Vesta as a function of the rotational phase.

rotational phases). The model predictions match the observed lightcurve amplitude as well as the lightcurve phase (in the observer's time frame). We also indicated the rotational phase where the Olbers feature (Zellner et al. 1997; Binzel et al. 1997) on Vesta's surface would be visible. We cannot see any influence of this surface feature with lower albedo. Müller \& Barnes (2007) presented a more detailed discussion on how various thermal properties of this surface feature would influence the thermal lightcurve variations at different wavelengths. They found strong emissivity variations which they attributed to the Olbers structure and a neighbouring region. The deviation from the model predictions at rotational phases between $240-280^{\circ}$ in Fig. 4 are also close to the Olbers region and might therefore be the far-IR signature of the ejecta material which is deposited on one side of the Olbers impact structure, as discussed in Müller \& Barnes (2007).

\section{Conclusions}

Via radiometric techniques we derived the first far-IR-based diameter and albedo values for the four large asteroids Ceres, Pallas, Vesta, and Hygiea. Based on photometry in the wavelength range $46-85 \mu \mathrm{m}$ we calculated effective diameters $D_{\text {eff }}$ of $960 \pm 17 \mathrm{~km}, 534 \pm 15 \mathrm{~km}, 549 \pm 13 \mathrm{~km}$, and $469 \pm 27 \mathrm{~km}$ for these four objects. The corresponding albedo values $p_{V}$ are $0.096 \pm 0.003,0.142 \pm 0.009,0.317 \pm 0.015$, and $0.056 \pm 0.006$, respectively. We found excellent agreement with direct measurements from HST (Ceres and Vesta) and speckle/occultation results (Pallas and Hygiea). Our data contain also the first far-IR lightcurve of an asteroid: Vesta's thermal lightcurve nicely follows the shape-introduced variations. Albedo and grain effects are important in the visual and mm-lightcurves, but seem to play a very small role at far-IR wavelengths.

The catalogue of LWS observations of asteroids contains photometric and spectroscopic measurements of the main representatives of the main asteroid belt. The data will remain useful for thermophysical and mineralogical studies of these bodies, especially in the context of the Dawn space mission to Ceres and Vesta.
Acknowledgements. We would like to thank Tim Grundy and his colleagues at RAL for preprocessing the non-standard data, and for the explanations concerning the peculiarities of these observations.

LIA is a joint development of the ISO-LWS Instrument Team at Rutherford Appleton Laboratories (RAL, UK - the PI Institute) and the Infrared Processing and Analysis Centre (IPAC/Caltech, USA).

The ISO Spectral Analysis Package (ISAP) is a joint development by the LWS and SWS Instrument Teams and Data Centers. Contributing institutes are CESR, IAS, IPAC, MPE, RAL and SRON.

\section{References}

Binzel, R. P., Gaffey, M. J., Thomas, P. C., et al. 1997, Icarus, 128, 95 Bowell, E., Hapke, B., Domingue, D., et al. 1989, in Asteroids II (Tucson: University of Arizona Press), 524

Carry, B., Kaasalainen, M., Dumas, C., et al. 2007, in AAS/Division for Planetary Sciences Meeting Abstracts, 39, \#30.08

Carry, B., Dumas, C., Fulchignoni, M., et al. 2008, A\&A, 478, 235

Clegg, P. E., Ade, P. A. R., Armand, C., et al. 1996, A\&A, 315, L38

Degewij, J., Tedesco, E. F., \& Zellner, B. 1979, Icarus, 40, 364

Dotto, E., Barucci, M. A., Müller, T. G., Storrs, A. D., \& Tanga, P. 2002, in Asteroids III, ed. W. F. Bottke Jr., A. Cellino, P. Paolicchi, \& R. P. Binzel, 219

Drummond, J., \& Christou, J. 2008, Icarus, 197, 480

Dunham, D. W., Dunham, J. B., Binzel, R. P., et al. 1990, AJ, 99, 1636

Gry, C., Swinyard, B., Harwood, A., et al. 2003, The ISO Handbook: LWS - The Long Wavelength Spectrometer, ESA SP-1262

Harris, A. W., \& Lagerros, J. S. V. 2002, in Asteroids III, ed. W. F. Bottke Jr., A. Cellino, P. Paolicchi, \& R. P. Binzel (Tucson: University of Arizona Press), 205

Henning, T., Il'In, V. B., Krivova, N. A., Michel, B., \& Voshchinnikov, N. V. 1999, A\&AS, 136, 405

Hormuth, F., \& Müller, T. G. 2006, Catalogue of LWS observations of asteroids, http://ida.esac.esa.int:8080/hpdp/technical_reports/ technote36.pdf

Kaasalainen, M., Mottola, S., \& Fulchignoni, M. 2002, in Asteroids III, ed. W. F. Bottke Jr., A. Cellino, P. Paolicchi, \& R. P. Binzel (Tucson: University of Arizona Press), 139

Kessler, M. F., Steinz, J. A., Anderegg, M. E., et al. 1996, A\&A, 315, L27

Lagerkvist, C.-I., Magnusson, P., Williams, I. P., et al. 1992, A\&AS, 94, 43

Lagerkvist, C. I., Piironen, J., \& Erikson, A. 2001, Asteroid Photometric Catalogue, Fifth Update, Uppsala Astron. Obs.

Lagerros, J. S. V. 1996, A\&A, 310, 1011

Lagerros, J. S. V. 1997, A\&A, 325, 1226

Lagerros, J. S. V. 1998, A\&A, 332, 1123

Lloyd, C. 2003, in The Calibration Legacy of the ISO Mission, ed. L. Metcalfe, A. Salama, S. B. Peschke, \& M. F. Kessler, ESA SP-481, 399

Marchis, F., Kaasalainen, M., Hom, E. F. Y., et al. 2006, Icarus, 185, 39

Millis, R. L., \& Dunham, D. W. 1989, in Asteroids II, ed. R. P. Binzel, T. Gehrels, \& M. S. Matthews, 148

Müller, T. G. 2002, Meteoritics \& Planetary Science, 37, 1919

Müller, T. G. 2003, in Exploiting the ISO Data Archive - Infrared Astronomy in the Internet Age, ed. C. Gry, S. B. Peschke, J. Matagne, P. García-Lario, R. Lorente, A. Salama, \& E. Verdugo, ESA SP-511, 47

Müller, T. G., \& Barnes, P. J. 2007, A\&A, 467, 737

Müller, T. G., \& Lagerros, J. S. V. 1998, A\&A, 338, 340

Müller, T. G., \& Lagerros, J. S. V. 2002, A\&A, 381, 324

Müller, T. G., Lagerros, J. S. V., Burgdorf, M., et al. 1999, in The Universe as Seen by ISO, ed. P. Cox \& M. F. Kessler, ESA-SP, 427, 141

Müller, T. G., Ábrahám, P., \& Crovisier, J. 2005, Space Sci. Rev., 119, 141

Ragazzoni, R., Baruffolo, A., Marchetti, E., et al. 2000, A\&A, 354, 315

Redman, R. O., Feldman, P. A., Matthews, H. E., Halliday, I., \& Creutzberg, F. 1992, AJ, 104, 405

Redman, R. O., Feldman, P. A., \& Matthews, H. E. 1998, AJ, 116, 1478

Salisbury, J. W. 1993, in Remote Geochemical Analysis: Elemental and Mineralogical Composition, ed. C. M. Pieters, \& P. A. J. Englert (New York: Cambridge University Press), 79

Shevchenko, V. G., \& Tedesco, E. F. 2006, Icarus, 184, 211

Tedesco, E. F., Egan, M. P., \& Price, S. D. 2002a, AJ, 124, 583

Tedesco, E. F., Noah, P. V., Noah, M., \& Price, S. D. 2002b, AJ, 123, 1056

Thomas, P. C., Binzel, R. P., Gaffey, M. J., et al. 1997, Icarus, 128, 88

Thomas, P. C., Parker, J. W., McFadden, L. A., et al. 2005, Nature, 437, 224

Torppa, J., Kaasalainen, M., Michalowski, T., et al. 2003, Icarus, 164, 346

Zellner, B. H., Albrecht, R., Binzel, R. P., et al. 1997, Icarus, 128, 83 